\title{
ANESTESIA DENTAL LOCAL SIN AGUJA: UNA REVISIÓN INTEGRATIVA DE LA LITERATURA.
}

\author{
Needle-free local anesthesia in dentistry: An integrative literature review
}

\author{
Alvarez-Sarmiento Jessica ${ }^{1}$, Ruiz-Proaño Andrea ${ }^{2}$, Álvarez-Vásquez José ${ }^{3 *}$. \\ ${ }^{1}$ Estudiante de quinto año de la Facultad de Odontología de la Universidad de Cuenca, Ecuador. \\ 2 Estudiante de quinto año de la Facultad de Odontología de la Universidad de Cuenca, Ecuador. \\ ${ }^{3}$ Esp. en Endodoncia. Director de la Especialidad en Endodoncia, Universidad de Cuenca, Ecuador \\ *jose.alvarezv@ucuenca.edu.ec
}

\begin{abstract}
Resumen
La anestesia local sigue siendo uno de los pilares de las técnicas de control del dolor en Odontología y, es ampliamente administrada empleando jeringuilla y aguja de inyección. Sin embargo, las técnicas anestésicas que no emplean aguja son también importantes elementos del arsenal odontológico para el control del dolor, aunque son poco conocidas y empleadas. El propósito de esta revisión es describir las técnicas anestésicas libres de aguja disponibles, así como sus dispositivos y perspectivas futuras. Se realizó una revisión integrativa de la literatura en el idioma inglés para evaluar las publicaciones de las últimas dos décadas, utilizando las bases de datos PubMed, Scopus, EBSCO, Science Direct y Wiley Online Library, empleando las siguientes palabras clave: anestesia dental local, anestesia sin aguja, anestesia dental a presión y anestesia intranasal. Se seleccionaron únicamente artículos y libros de mayor relevancia. Finalmente, se obtuvo 33 artículos y 1 libro. Las técnicas de anestesia dental sin aguja disponibles actualmente son: anestesia a presión, intrasulcular e intranasal, todas ellas emplean dispositivos diferentes y poseen aplicaciones, ventajas, desventajas y limitaciones inherentes que podrían ser mejoradas o superadas en un futuro próximo.
\end{abstract}

Palabras clave: anestesia dental local, anestesia sin aguja, anestesia dental a presión, anestesia intranasal.

\begin{abstract}
Local anesthesia remains the backbone of pain control techniques in dentistry and is predominantly administered by injection using a syringe and needle. Nevertheless, needle-free local anesthesia techniques are also important adjuncts in the pain control armamentarium in dentistry, although they are not well known or widely used. The purpose of this review was to identify the available needle-free anesthetic techniques, to describe the devices used for administration, and to discuss future trends in the field. An English language integrative literature review was performed to assess publications over the last two decades using PubMed, Scopus, EBSCO, Science Direct, and Wiley Online Library databases based on the following keywords: local dental anesthesia, needleless anesthesia, pressure dental anesthesia, and intranasal anesthesia. Only articles and books of greater relevance were selected. Finally, 33 articles and one book were assessed. The currently available needleless dental anesthesia techniques rely on jet injectors, as well as intrasulcular and intranasal anesthesia, all of which employ different devices with their own inherent applications, advantages, disadvantages, and limitations that may be improved upon or overcome in the near future.
\end{abstract}

Key words: local dental anesthesia, needleless anesthesia, pressure dental anesthesia, intranasal anesthesia.

\section{Introducción}

La anestesia local dental sigue siendo uno de los pilares de las técnicas de control del dolor, siendo ampliamente administrada mediante jeringuilla y aguja de inyección ${ }^{1}$ e irónicamente esta técnica es vista por muchos pacientes como el único aspecto doloroso de la cita dental ${ }^{2}$. Por otro lado, las técnicas anestésicas que no emplean aguja, son también parte del arsenal odontológico para el control del dolor, pero son poco conocidas y empleadas y, hasta donde nosotros sabemos, no existe en la literatura una revisión integrativa actualizada de éstas técnicas. El miedo a las inyecciones dolorosas, y el subsecuente comportamiento de evitar la consulta, son barreras significativas en el cuidado de la salud bucal ${ }^{3}$, ya que millones de personas evitan visitar al odontólogo anualmente debido al miedo por la penetración de la aguja ${ }^{4}$. El miedo a las agujas es llamado tripanofobia ${ }^{1}$ 
Es importante destacar que el Acta de Prevención y Seguridad de Pinchazos por Aguja de la ADA (Asociación Dental Americana) dispone que se emplee tecnología libre de aguja siempre que sea posible ${ }^{5}$, y en este sentido, todas las técnicas de anestesia dental sin aguja tienen la ventaja inherente de no emplearla, por lo que disminuyen el riesgo ocupacional de transmisión de patógenos por vía sanguínea debido al empleo de agujas, y deben ser siempre incentivadas y promovidas $^{6}$.

En la presente revisión, se abordarán las 3 técnicas anestésicas locales sin empleo de aguja, referidas hasta el momento en Odontología, esto es, anestesia a presión, intrasulcular e intranasal. No se incluirá la anestesia electrónica, pues per se no constituye un método anestésico ya que no emplea ningún tipo de anestésico local, ni tampoco lo referente a los anestésicos tópicos.

\section{Estado del Arte}

El presente estudio es una revisión integrativa de la literatura, por lo que se incluye estudios de tipo experimental y no experimental, con el fin de abordar diversos aspectos como conceptos, definiciones, metodologías y evidencia científica disponible $^{7}$. Se realizó una búsqueda electrónica de artículos y libros científicos en el idioma inglés utilizando las bases de datos PubMed, Scopus, EBSCO, Science Direct y Wiley Online Library. Los términos clave de búsqueda empleados en combinación fueron "anestesia dental local", "anestesia sin aguja", "anestesia dental a presión” y "anestesia intranasal”. La búsqueda se realizó en artículos publicados desde enero de 2000 hasta diciembre de 2019. En la mayoría de los casos, se omitieron los artículos que no tenían un enlace explícito a las palabras clave buscadas. Luego, se procedió a remover duplicados. Después de la selección inicial basada en títulos y resúmenes, solo se seleccionaron artículos y libros de mayor relevancia. Los artículos se clasificaron de acuerdo con las secciones de esta revisión.

Finalmente, se evaluó el texto completo de 33 artículos seleccionados, así como la información pertinente de un libro, para proporcionar a los lectores la información más reciente y relevante sobre las técnicas de anestesia dental local que no requieren una aguja.

\section{ANESTESIA DENTAL A PRESIÓN}

Los sistemas a presión fueron los primeros sistemas introducidos de anestesia dental sin aguja. En 1933 se diseñó el primer prototipo de jeringa y, desde entonces, se ha perfeccionado para hacer posible su aplicación en Odontología ${ }^{8}$. En 1958 la primera publicación relacionada con esta técnica reveló una tasa de éxito significativa (87\%) para procedimientos restaurativos y exodoncias ${ }^{9}$.

La anestesia mediante un dispositivo de inyección de chorro, se consigue presurizando y acelerando la solución anestésica a alta velocidad $(>100 \mathrm{~m} / \mathrm{s})$ a través de un orificio estrecho, creando un chorro de fluido fino que penetra fácilmente en piel y mucosas ${ }^{8},{ }^{10}$ La alta presión ejercida en el dispositivo hace que la solución anestésica sea inmediatamente absorbida por la vaina de mielina de las fibras nerviosas ${ }^{11}$. Se ha referido que el paciente siente un "golpecito de gas" en la mucosa, que es como chasquear el dedo contra su piel ${ }^{12}$.

\section{Componentes de un dispositivo de inyección a presión}

Los sistemas de inyección a presión sin aguja constan de tres componentes principales (Fig. 1): a) un dispositivo de inyección con una ampolla que contendrá el fármaco, luego de que este es transferido desde los cartuchos anestésicos tradicionales, b) una boquilla que está en contacto con la piel, a través de la cual se transporta el medicamento y, c) una fuente de presión que mediante métodos mecánicos en un resorte almacena energía suficiente, que es liberada al accionar un gatillo y, finalmente el resorte empuja al émbolo, que impulsará el anestésico a través de la boquilla ${ }^{12}$.

\section{Mecanismo de un dispositivo de inyección a presión}

En estudios que utilizan modelos de gel de poliacrilamida, se ha demostrado que la penetración del anestésico se produce en tres etapas distintas (Fig. 2): erosión, estancamiento y dispersión ${ }^{13}$. Durante la erosión, se produce un agujero sobre la superficie mucosa y posteriormente el impacto continuo del chorro da lugar a un canal introductorio cilíndrico de fluido, que alcanza un punto de estancamiento, para finalmente dispersarse de manera circular ${ }^{13-15}$.

\section{Sistemas de anestesia dental a presión}

Los dispositivos a presión más utilizados en Odontología son INJEX, MadaJet XL y Comfort-in (Fig. 3), los cuales se describen a continuación, destacando el hecho de que ninguno de ellos funciona con el cartucho anestésico dental tradicional, sino que emplean sus propias ampollas de inyección. El dispositivo Syrijet Mark II, era uno de los favoritos en el área odontológica, pero actualmente ya no se lo fabrica, por tal motivo ha sido excluido de la presente revisión ${ }^{1}$.

INJEX. El sistema INJEX utiliza una ampolla de inyección, de un solo uso, que posee una boquilla con un micro orificio de tan solo $0.17 \mathrm{~mm}^{15}$. El dispositivo, de acero inoxidable, es del tamaño de un bolígrafo y de peso ligero (aproximadamente $75 \mathrm{~g})^{8}$. Se coloca firmemente sobre la mucosa oral correspondiente al ápice del diente, formando un ángulo de $90^{\circ}$ (Fig. 3-A). Después de presionar 


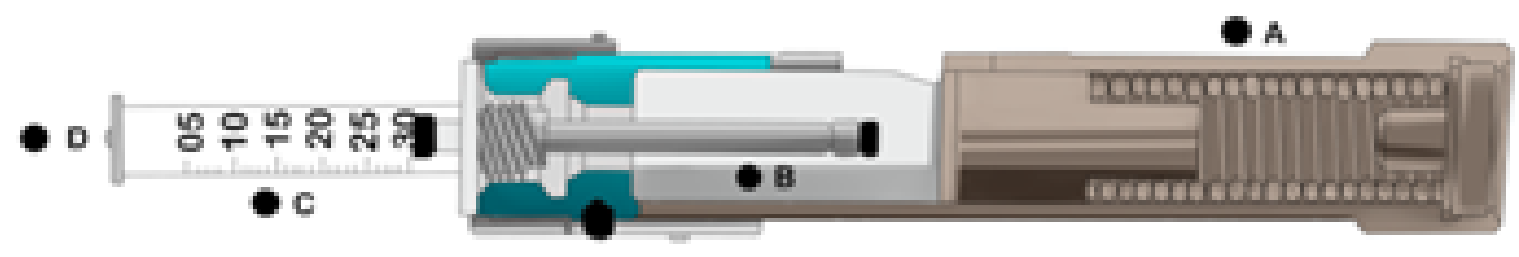

Fig. 1. Componentes de un sistema de inyección a presión. A: resorte. B: émbolo. C: ampolla que contiene el fármaco. D: boquilla.

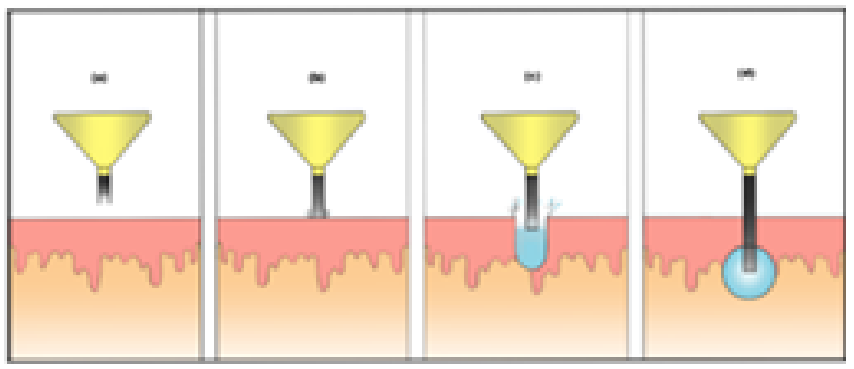

Fig. 2. Mecanismo de penetración del anestésico en la mucosa mediante dispositivos a presión

ligeramente el gatillo, el medicamento (máximo $0.3 \mathrm{ml}$ por disparo, para este dispositivo) se administra con una presión de 3000 psi (libras de fuerza por pulgada cuadrada) a los tejidos cutáneos/subcutáneos, a una profundidad de 5-8 $\mathrm{mm}$, en menos de 2 segundos ${ }^{8,16,17}$.

MADAJET XL. También funciona mediante el uso de presión (1800 psi) para descargar el anestésico ${ }^{18}$. Un compartimento de vidrio reutilizable y autoclavable puede contener hasta $4 \mathrm{ml}$ de solución anestésica ${ }^{11,19}$. Cada inyección administra a través de un orificio de $0.12 \mathrm{~mm}$ de diámetro y a una profundidad de 2 a $2.5 \mathrm{~mm}^{11}$ un volumen de $0.1 \mathrm{ml}$ de solución anestésica ${ }^{11,}{ }^{20} \mathrm{El}$ segmento de suministro de anestesia en este sistema forma un ángulo de 45 grados con el cuerpo principal del dispositivo (Fig. 3-B), lo que permite un posicionamiento más fácil y un mejor contacto de la punta del dispositivo con la superficie tisular, que puede resultar en menor presión durante la administración del anestésico y menos posibilidades de fuga, que causa un sabor desagradable ${ }^{17}$.

COMFORT-IN. Utiliza una ampolla de inyección de un solo uso, posee un orificio de $0.15 \mathrm{~mm}$, el cual crea un chorro de fluido fino con dosis que se puede calibrar desde $0.1 \mathrm{~mL}$ a un máximo de $0.3 \mathrm{ml}$ de solución anestésica, la cual penetra en la piel en menos de un tercio de segundo. Similar al dispositivo INJEX, el ángulo de administración es de $90^{\circ}$ con respecto a la mucosa $a^{21}$ (Fig. 3-C).

\section{USOS EN ODONTOLOGÍA DE SISTEMAS DE ANESTESIA DENTAL A PRESIÓN}

\section{Cirugía Oral}

En cirugía oral con frecuencia se deposita el anestésico local en múltiples sitios con fines de vasoconstricción y analgesia postoperatoria, lo cual es posible conseguir con un mínimo de esfuerzo y tiempo mediante el inyector a presión. También, es recomendado para la remoción de barras en arco, alambres de ligadura o para biopsias de punción pequeña ${ }^{23}$.

Con referencia a extracciones dentales en adultos, la eficacia de los dispositivos a presión es contradictoria. Existen dos estudios, en los cuales se comparó la efectividad de la técnica con aguja e INJEX. En uno de ellos se concluyó que, durante la extracción el dolor percibido por el paciente al emplear el sistema a presión fue mayor, y el $28.5 \%$ necesitó anestesia adicional con la técnica tradicional para finalizar la extracción. En este estudio, se evidenció que INJEX no es efectivo para este tipo de procedimientos ${ }^{16}$.

Por el contrario, en otro estudio se reporta una tasa de éxito alta para los dispositivos a presión, 92.6\% para el maxilar y $85.2 \%$ para la mandíbula. Además, la preferencia también fue significativamente mayor $(77.7 \%)$. Los hallazgos discrepantes entre estos dos estudios pueden deberse al hecho de que el volumen de anestésico utilizado en éste estudio fue tres veces mayor respecto al primer estudio mencionado anteriormente ${ }^{22}$.

\section{Odontopediatría}

Dos estudios que compararon la eficacia anestésica de INJEX y MadaJet XL vs la técnica tradicional, encontraron resultados significativamente diferentes entre ambos sistemas sin aguja, lo que podría obedecer a la diferente angulación de administración de cada dispositivo, siendo MadaJet XL mejor en este sentido ${ }^{11},{ }^{17}$

En el estudio que usó MadaJet XL, además de proporcionar comodidad al paciente pediátrico durante la administración anestésica, se encontró que su uso era apropiado para todos los procedimientos en dientes primarios. Se aplicó con éxito para extracciones, terapia 


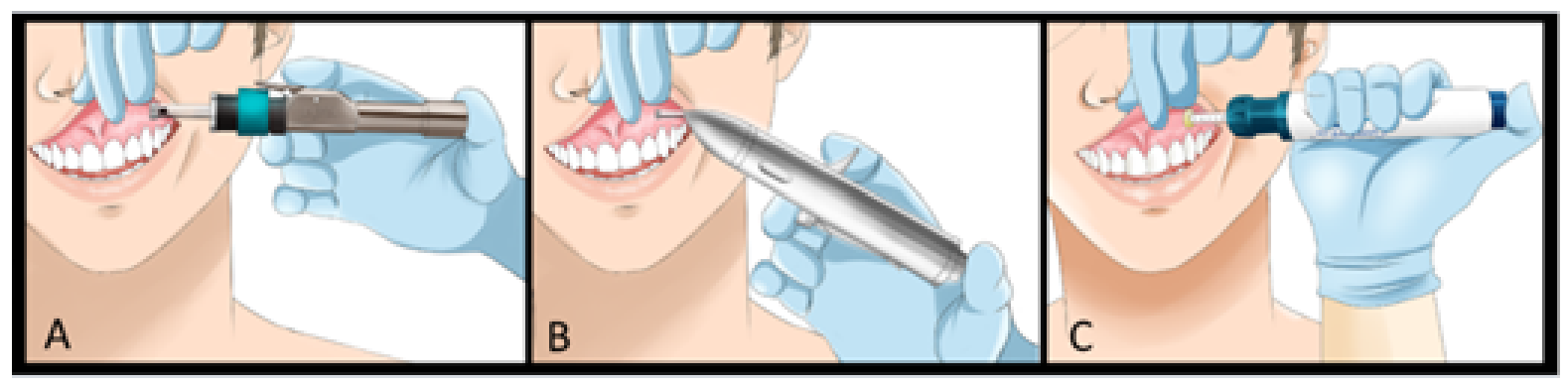

Fig. 3. Sistemas de anestesia a presión. Nótese la diferente angulación requerida para la administración anestésica. A: INJEX forma un ángulo de $90^{\circ}$ con respecto a la mucosa. B: MadaJet XL forma un ángulo de $45^{\circ}$ entre el segmento de suministro de anestesia con respecto al cuerpo principal. C: Comfort-in $\left(90^{\circ}\right)$

pulpar, preparaciones cavitarias y procedimientos clínicos diversos (drenaje de abscesos, sutura, colocación de grapas de diques de goma), con tasas de éxito del 96-100\%11. Por otro lado, en el estudio que usó INJEX, se informó un mayor número de experiencias negativas, y la gran mayoría de los niños expresaron su preferencia por la aguja. Además, después de usar INJEX, el $80.5 \%$ requirió anestesia adicional para completar el procedimiento sin dolor; se requirió anestesia adicional para todas las extracciones, y para gran parte de las restauraciones. En este estudio no se realizó terapia pulpar u otros procedimientos como en el estudio descrito anteriormente. ${ }^{17}$

\section{Periodoncia}

Una de las desventajas de la anestesia con aguja es el largo período de entumecimiento en los tejidos blandos, lo que se elimina con el uso de sistemas sin aguja ${ }^{8,}{ }^{19} \mathrm{Si}$ bien no se ha realizado un estudio comparativo de las técnicas convencionales y sin aguja, se ha demostrado que los dispositivos a presión permiten completar con comodidad el RAR, con una mayor tasa de éxito para el alivio del dolor en comparación con una mezcla eutéctica de agentes anestésicos locales administrada tópicamente (EMLA). Pese a esto, la mayoría de los pacientes prefirió la aplicación de EMLA, que se atribuyó a una experiencia menos traumática, ya que el aspecto voluminoso del dispositivo MadaJet XL causó aprensión en los pacientes ${ }^{19}$. Por otro lado, el uso de dispositivos a presión para procedimientos de gingivectomía también se ha citado en la literatura ${ }^{20}$.

\section{Operatoria Dental y Prostodoncia}

Procedimientos restauradores simples, como clase I y II se pueden completar cómodamente con anestesia a presión, siempre que el procedimiento se finalice en 20 a 25 minutos, lo cual puede estar supeditado a la habilidad y experiencia del operador ${ }^{20,} .{ }^{24}$ Este sistema también podría usarse para la colocación de hilos de retracción, de grapas para dique de goma y para la cementación de coronas y carillas ${ }^{20}$.

\section{Ortodoncia}

Los dispositivos sin aguja proporcionan adecuada anestesia en los tejidos blandos, lo que sería útil para colocar bandas de ortodoncia, mantenedores de espacio $^{25}$ e incluso miniimplantes en zonas anteriores ${ }^{26}$. Al accionar el dispositivo se crea un área eritematosa superficial de 2-3 mm, que puede guiar y facilitar la colocación del mini implante. Por tanto, los ortodoncistas que realizan procedimientos coadyuvantes mínimamente invasivos, como el anclaje esquelético, pueden beneficiarse de esta técnica ${ }^{26}$.

\section{Endodoncia}

Se debe enfatizar que, en la literatura no se ha reportado casos de tratamiento endodóntico en adultos, realizados bajo anestesia local a presión. Sin embargo, en base a los estudios en los que se calculó la duración de la anestesia pulpar con ayuda de un pulpómetro eléctrico, se concluye que el sistema sin aguja es insuficiente para completar un procedimiento de esta complejidad, ya que no proporciona anestesia adecuada más allá de los 25 minutos $^{8,20,24}$. Por otro lado, se ha referido que esta técnica podría ser útil para procedimientos de incisión y drenaje de abscesos. ${ }^{20}$

\section{Ventajas y desventajas}

La anestesia a presión ofrece ciertas ventajas, como permitir su uso en pacientes con fobia a las agujas, ${ }^{12}$ es rápida y fácil de usar, genera menos estrés tanto para el paciente como para el profesional, el dispositivo es autoclavable-reutilizable (rentabilidad), ${ }^{11}$ menor daño a los tejidos, ausencia de accidentes y transmisión de enfermedades por pinchazo de aguja $\mathrm{y}$, evita lesiones autoinfligidas por entumecimiento postoperatorio. ${ }^{23}$ En esta técnica es prácticamente imposible originar una sobredosis, debido a que la cantidad de anestésico administrado es limitada y su efecto localizado, lo que también resulta ser conveniente para niños de temprana edad o pacientes con trastornos sistémicos. ${ }^{20}$

Las principales desventajas son el costo inicial del 
equipo, posibilidad de hematomas residuales, apariencia voluminosa del dispositivo en el caso del MadaJet $\mathrm{XL}^{25}$ y sangrado en el sitio de inyección. ${ }^{8,17,20}$ Estos dispositivos también tienen el potencial de asustar a los pacientes con el ruido repentino y la sensación de presión que se produce al administrar el anestésico, ${ }^{8,16,17,20,25}$ especialmente si el dispositivo es usado en niños, puesto que es difícil demostrar y explicar la presión y el ruido exacto que se producirá. ${ }^{17}$ Además, la mayoría de los clínicos no están familiarizados con su uso $^{20}$ y solo se puede administrar un pequeño volumen por inyección, ${ }^{23}$ por lo que no se puede usar para realizar bloqueos nerviosos, y sólo es posible la infiltración y la anestesia superficial. ${ }^{27}$ Por otra parte, los sistemas INJEX y Comfort-in, durante su aplicación deben formar un ángulo de $90^{\circ}$, lo cual es difícil conseguir, particularmente en las superficies palatinas/ linguales y en el sector posterior, dando como resultado fugas y un sabor desagradable. ${ }^{16,17}$

\section{ANESTESIA DENTAL INTRASULCULAR}

Esta técnica incluye un pequeño dispositivo de plástico de reciente introducción llamado NumBee, capaz de adaptarse a cualquier jeringa carpule dental estándar. ${ }^{28} \mathrm{El}$ diseño innovador de NumBee consta de una hoja muy delgada de plástico, incluso más delgada que los palillos de dientes, en cuyo interior presenta una cánula de acero redondeada encargada de transportar el líquido anestésico. En el extremo de la hoja hay una pequeña punta redondeada, por donde emerge el líquido anestésico hacia el surco gingival ${ }^{28}$ (Fig. 4-A).

\section{Mecanismo de aplicación}

Se coloca a través del surco gingival, a la misma profundidad que ingresarían las cerdas de un cepillo dental y por la pequeña punta redondeada saldrá el líquido anestésico bajo presión (Fig. 4-B). Su característica importante es que no hay perforación de ningún tejido, ya que no posee punta afilada, lo que constituye una ventaja inherente para pacientes con fobia a las agujas. ${ }^{28}$

Debido a la falta de literatura sobre la eficacia y seguridad de su uso, se recomienda realizar estudios que permitan evaluar el éxito anestésico con este dispositivo durante procedimientos dentales, tanto en adultos como en niños. Sin embargo, llama la atención que el sitio web de este dispositivo ya no es accesible desde el año 2019.

\section{ANESTESIA DENTAL INTRANASAL}

La anestesia dental intranasal es uno de los últimos avances en el control del dolor en Odontología y, a pesar de haber sido aprobada por la FDA (Food and Drug Administration) en Junio del 2016 ${ }^{1,29-31}$ y, estar varios años en el mercado, muchos profesionales la desconocen. ${ }^{31}$ Kovanaze es el primer y único compuesto de aplicación intranasal usado para generar anestesia dental maxilar, el cual contiene una combinación de tetracaína (anestésico local) y oximetazolina (vasoconstrictor), compuestos que han sido usados durante varios años en medicina. ${ }^{1,6,32,34}$

Esta técnica fue un hallazgo casual por parte del Dr. Mark Kollar, mientras realizaba la atención rutinaria en pacientes del área de Otorrinolaringología, a quienes colocaba una pulverización de oximetazolina para proporcionar hemostasia y seguido tetracaína en gel para controlar el dolor. Se manifestaron reportes anecdóticos de los pacientes de sensación diferente o de "entumecimiento" en los dientes anterosuperiores ${ }^{1,} .{ }^{34}$ Desde entonces, diversos estudios se realizaron con el fin de evaluar la seguridad y eficacia de los compuestos para conseguir anestesia dental, considerando adecuada la concentración de la tetracaína al $3 \%$ con oximetazolina al $0.5 \%^{34}$. El clorhidrato de tetracaína es un anestésico local de tipo éster con alta potencia y efecto de larga duración, es degradado por estereasas plasmáticas, convirtiéndose rápidamente en un metabolito, el ácido p-butil aminobenzoico (PBBA), el cual es detectable fácilmente en el plasma sanguíneo ${ }^{29,32,35}$ - La oximetazolina es un agonista adrenérgico $\alpha 1$ y 2 , su actividad simpaticomimética estimula los receptores adrenérgicos produciendo vasoconstricción, reducción del flujo sanguíneo y además ayuda a ralentizar la absorción sistémica de la tetracaína y prolongar sus concentraciones en el tejido ${ }^{29,35}$. Es importante mencionar que la adrenalina es raramente usada de manera intranasal, ya que los efectos cardiovasculares son significativos por esta vía ${ }^{36}$.

\section{Distribución anatómica intranasal del anestésico}

Una característica importante de la anatomía ósea del maxilar superior es ser esponjosa y más porosa, especialmente el sector de los alveolos dentales, así como también la alta vascularización de las fosas nasales. Ambas variaciones anatómicas contribuyen a que el anestésico se absorba y distribuya rápidamente, alcanzando las fibras nerviosas del nervio alveolar superior anterior (ASA), que inerva a los dientes anterosuperiores, y el nervio alveolar superior medio (MSA), a premolares superiores ${ }^{1,31,33}$

Algunos estudios reportan una disminución del éxito anestésico a nivel de segundos premolares superiores, debido a una diferencia anatómica en su inervación. En su gran mayoría estos dientes están inervados por el nervio alveolar superior medio, pero se ha reportado que entre 28 y $54 \%$ de la población esta rama está ausente, y en este caso, la inervación pulpar proviene del plexo formado por ramificaciones del nervio alveolar superior posterior (PSA) $\mathrm{y}$ anterior $(\mathrm{ASA})^{1,30,37}$. Además, se podría atribuir a que la solución anestésica local es incapaz de llegar a las zonas posteriores del seno maxilar, es decir, a los dientes superiores más posteriores ${ }^{32,37}$. La eficacia anestésica también pueden verse afectada por los cambios de volumen óseo sinusal 


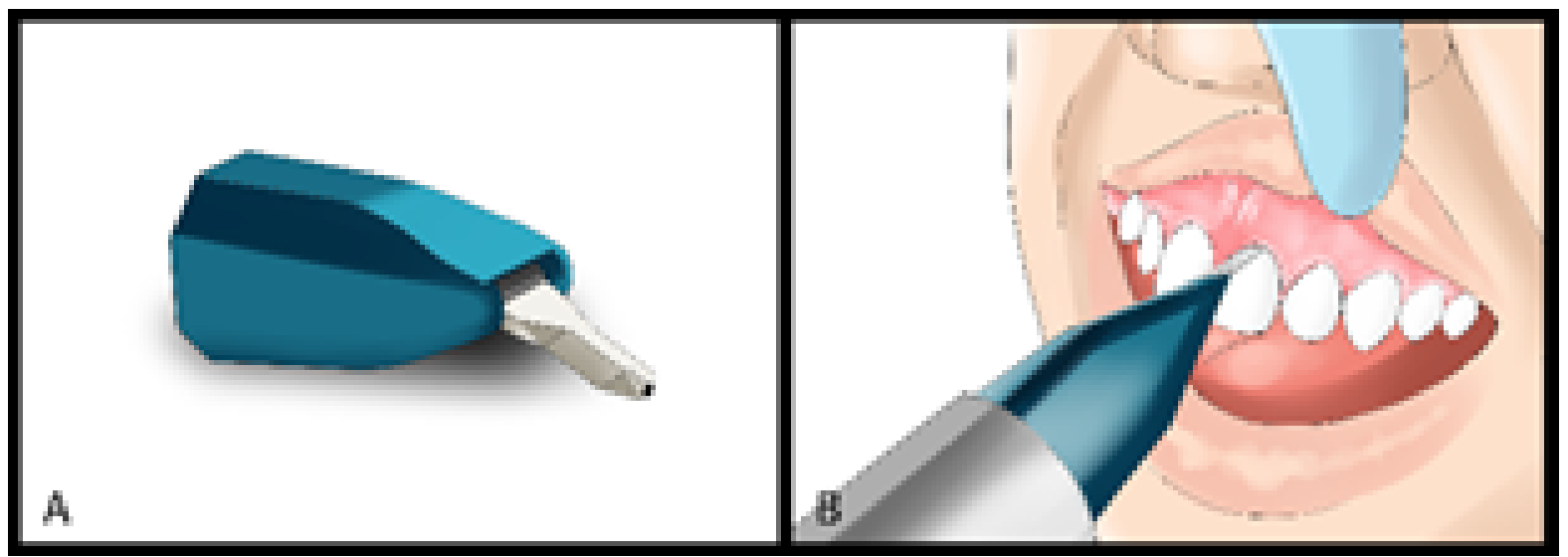

Fig. 4. A: diseño del dispositivo NumBee. B: mecanismo de aplicación del sistema NumBee en el surco gingival.

relacionados con la edad (neumatización del seno maxilar) ${ }^{33}$

\section{Dosificación del anestésico intranasal}

Kovanaze se emplea en el dispositivo descartable BD Acusspray ${ }^{32-35,37,38}$, el cual crea una pulverización al forzar el líquido a través de un atomizador de remolino de presión cuando se acciona la barra que contiene al émbolo ${ }^{39}$ (Fig.5).

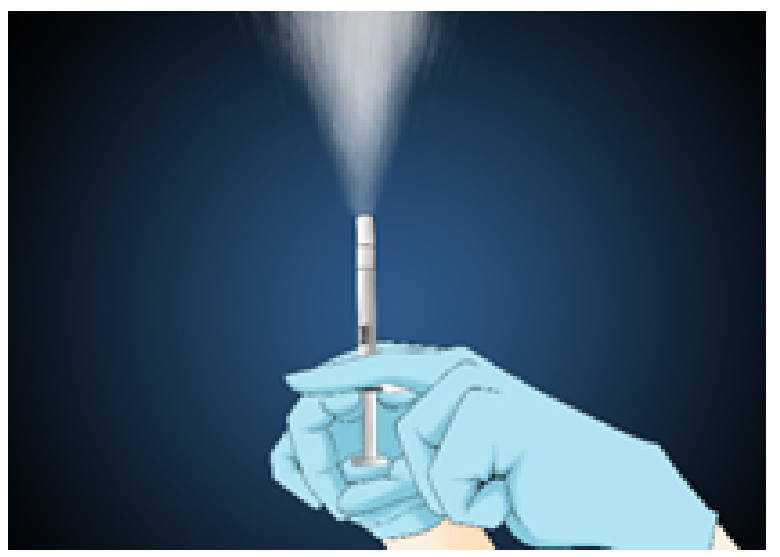

Fig. 5. Sistema de pulverización intranasal BD Accuspray crea el aerosol anestésico

Cada pulverización de $0.2 \mathrm{~mL}$ contiene $6 \mathrm{mg}$ de clorhidrato de tetracaína y $0.1 \mathrm{mg}$ de clorhidrato de oximetazolina, equivalente a $5.27 \mathrm{mg}$ de tetracaína y $0.08 \mathrm{mg}$ de oximetazolina. El contenido también incluye ácido cítrico, citrato de sodio, hidroxietilcelulosa, alcohol bencílico y agua, así como hidróxido de sodio y/o ácido clorhídrico para ajustar el $\mathrm{pH}$ de la solución, que es de $6.0 \pm$ $1.0^{29}$.

En adultos mayores de 18 años y en niños que pesan más de $40 \mathrm{~kg}$ se administra 2 pulverizaciones intranasales de $0.2 \mathrm{ml}$, con una separación de 4 a 5 minutos, después de la segunda pulverización se espera 10 minutos para iniciar el procedimiento dental. Únicamente en pacientes mayores a 18 años, se puede administrar una tercera pulverización $(0.2 \mathrm{ml})$ cuando no se ha logrado el éxito anestésico y, nuevamente esperar 10 minutos para empezar el procedimiento dental ${ }^{29}$.

\section{Ubicación del dispositivo intranasal}

El paciente debe estar erguido, mirando al frente y se coloca el dispositivo en la fosa nasal ipsilateral del diente a tratar, presionando el émbolo tan fuerte y rápido como sea posible en un solo movimiento ${ }^{29}, 30$

En la primera pulverización, se debe seguir una línea imaginaria entre el punto nasion y el subnasal, de tal manera que forme un ángulo aproximadamente de $90^{\circ}$ con el dispositivo intranasal, logrando alcanzar la zona del meato inferior (Fig. 6-A). Para la segunda pulverización, se toma como la misma línea imaginaria, pero formando un ángulo de aproximadamente $45^{\circ}$ respecto al dispositivo (Fig. 6-B), alcanzando la zona del meato medio ${ }^{29,40}$.

La correcta ubicación y angulación del dispositivo intranasal pueden contribuir al éxito anestésico, pues, en un estudio se menciona que la colocación del pulverizador en el interior de la vía aérea nasal principal obtiene mejores resultados de eficacia anestésica en comparación con la colocación a la entrada de la fosa nasal ${ }^{40}$.

\section{Usos en odontología de la anestesia dental intranasal}

\section{Operatoria dental}

El uso de Kovanaze se recomienda básicamente para procedimientos dentales poco invasivos como restauraciones dentales que no comprometan a la pulpa dental. ${ }^{37}$ El éxito anestésico es considerado cuando se completa dicho 


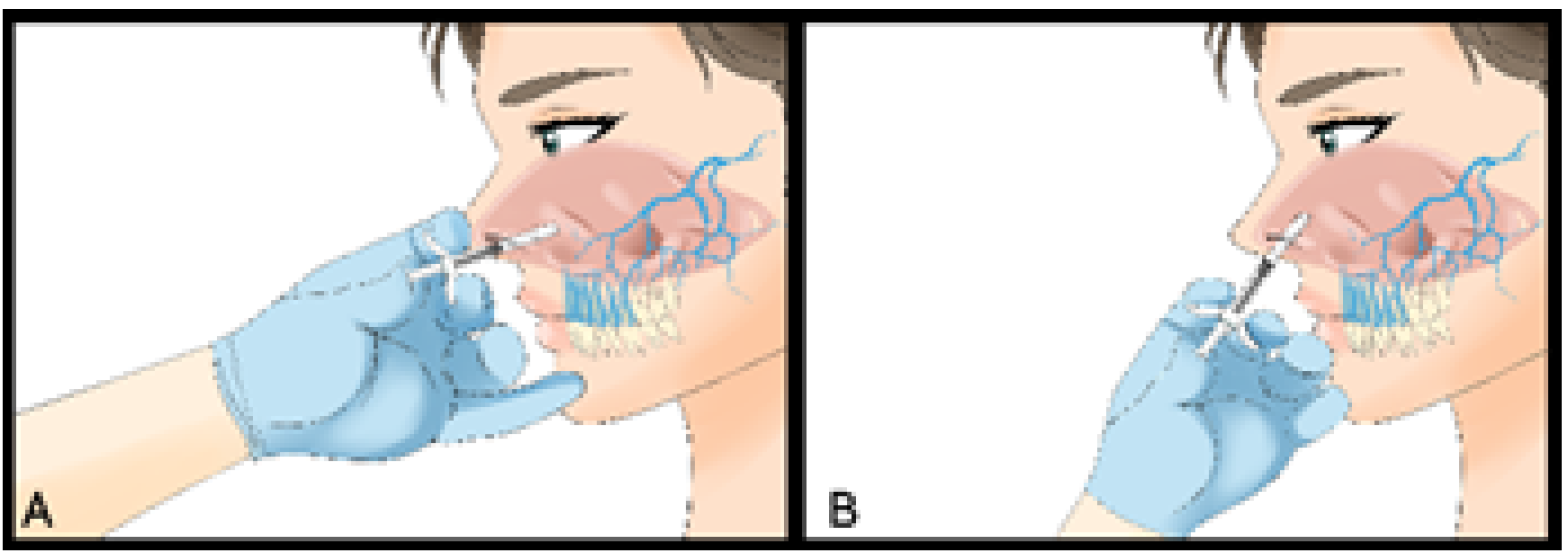

Fig. 6. A: primera pulverización a una angulación de $90^{\circ}$ del dispositivo intranasal. B: Segunda pulverización con la punta del pulverizador colocada aproximadamente a $45^{\circ}$.

procedimiento sin necesidad de administrar anestesia de rescate; en la mayoría de estudios realizados se reporta una tasa de éxito entre 83 y $90 \%$ para dientes anterosuperiores, ${ }^{6,33,34,37}$ sin embargo, este disminuye en segundos premolares entre 60 y $66 \%$, en razón de su variabilidad anatómica de inervación ${ }^{37}$. No obstante, en un reciente estudio se reportó menor éxito para Kovanaze intranasal entre 22 y $37 \%$, comparada con el 89 y $91 \%$ de la infiltración inyectable de lidocaína ${ }^{30}$.

\section{Odontopediatría}

En un estudio de fase 3 en niños de 3 a 17 años, la variable principal del éxito anestésico fue la realización de un procedimiento dental convencional (extracción de caries y restauración de un diente) sin la necesidad de anestesia de rescate. En este estudio, fue evidente que la tasa de éxito dependía del peso del niño, ya que la administración correspondiente de Kovanaze y placebo fue similar (88\%) en pacientes con un peso de $10-20 \mathrm{~kg}$. No se observaron diferencias significativas en aquellos que pesaban entre $20 \mathrm{y}$ $40 \mathrm{~kg}$ (58\% para Kovanaze vs. $42 \%$ para placebo), mientras que hubo una diferencia significativa en niños que pesaban $40 \mathrm{~kg}$ o más $(90 \% \text { vs. } 40 \% \text {, respectivamente })^{41}$.

Otro estudio clínico de fase 2 en niños está en ejecución, con el fin de evaluar la eficacia de Kovanaze en la anestesia pulpar. Sin embargo, este estudio se suspendió temporalmente porque el fabricante informó que Kovanaze no está disponible por el momento y que no estaría durante los próximos 6 meses $^{42}$.

Por otro lado, se ha referido que el uso de cualquier medicamento intranasal en niños menores de 5 años no tiene buenos resultados debido al subdesarrollo o taponamiento de las vías aéreas principales, lo cual no permitiría la difusión correcta del medicamento $^{40}$

Cirugía, periodoncia, ortodoncia, prostodoncia y endodoncia

No existe evidencia en la literatura sobre estos procedimientos dentales, y todos los estudios arriba citados solo evaluaron la eficacia y seguridad de kovanaze intranasal en procedimientos dentales restaurativos en el sector anterosuperior.

\section{Ventajas y desventajas}

La anestesia intranasal es una técnica sin aguja que permite la infiltración anestésica local y reduce el grado de ansiedad, miedo y dolor 43. La administración de un volumen reducido de kovanaze $(0,4$ o $0,6 \mathrm{ml})$ puede lograr la anestesia suficiente en dientes anterosuperiores para completar los procedimientos restaurativos ${ }^{29,31,34}$, y por ende será poco probable que ocurran reacciones de sobredosis al fármaco ${ }^{32}$. Otra ventaja es la falta de anestesia extraoral de tejidos blandos, ya que únicamente se anestesia la zona palatina anterior, por tanto no se aprecia la misma sensación de entumecimiento y hormigueo que perdura por varias horas como ocurre con la técnica infiltrativa tradicional con aguja ${ }^{1,}{ }^{29}$ lo que puede ser ventajoso en caso de procedimientos de restauración estéticos en la zona anterior.

Entre sus desventajas podemos mencionar que el costo es relativamente mayor en comparación con la técnica anestésica convencional, tiene largo periodo para iniciar su acción, que es de 14 a 24 minutos, por lo que se requiere una cita más larga para llevar a cabo el procedimiento dental ${ }^{30}$.

\section{Efectos adversos}

Las reacciones adversas leves más comunes ocurren en el 
$10 \%$ de los pacientes, probablemente son inducidas por la oximetazolina e incluyen: secreción, irritación, ulceración, congestión, molestias nasales, dolor de garganta, dolor de cabeza, lagrimeo $6,30,33-35,37$ y un ligero incremento de la presión arterial, los cuales regresan a la normalidad horas más tarde $\mathrm{e}^{6,35,37}$; un estudio también reportó congestión sinusal $^{30}$, así como disminución del umbral olfatorio ${ }^{6,37}$ . Además, se ha reportado que puede existir cierto riesgo de metahemoglobinemia, especialmente en pacientes con metahemoglobinemia congénita o idiopática. ${ }^{29}$ También, el caso de un paciente con antecedentes de tiroiditis linfocítica, en quien se incrementó la presión arterial luego de administrar Kovanaze, la misma que se normalizó horas después $^{33}$.

\section{Contraindicaciones}

Está contraindicado en pacientes con hipertensión no controlada, enfermedad tiroidea activa, pacientes con antecedentes de alergia a la tetracaína o anestésicos locales de tipo éster y a la oximetazolina, y también en pacientes con antecedentes de hemorragia nasal frecuente, personas que usan inhibidores de la monoaminooxidasa, antagonistas beta adrenérgicos no selectivos o antidepresivos tricíclicos, ya que puede causar hipertensión ${ }^{29}$.

\section{Discusión}

Las técnicas de manejo anestésico sin aguja han conseguido avances significativos en la odontología, cambiando las perspectivas de los pacientes sobre los procedimientos dentales, por lo que es importante que los odontólogos estén familiarizados con todas las actualizaciones tecnológicas para optimizar la comodidad del paciente ${ }^{32}$.

En el caso de la anestesia dental a presión, el tipo de dispositivo parece influir en la efectividad de la anestesia. La efectividad de MadaJet XL varió de 88-100\%11,19, 20 mientras que solo un estudio informó sobre el uso del dispositivo Comfort-in en el que la anestesia de rescate no era necesaria, lo que sugiere una tasa de éxito del $100 \%^{24}$. Para INJEX, la eficacia disminuye entre 19 a $71 \%$, teniendo en cuenta que todos estos porcentajes varían dependiendo del procedimiento realizado, como ya se describió anteriormente ${ }^{16,17}$. No obstante, para contrastar esta última cifra existe un estudio que reporta una tasa de éxito de hasta 92\%; el incremento en este caso podría atribuirse al volumen total inyectado, ya que fue el triple del que otros autores han utilizado con este mismo dispositivo ${ }^{22}$. Aun así, ninguno de los dispositivos a presión ha logrado superar a la técnica tradicional, que consigue una anestesia más profunda, incluso utilizando la misma cantidad de solución anestésica que los sistemas libres de aguja ${ }^{16,17,20,22,24}$. En la literatura, por un lado, se ha informado que el volumen influye de manera positiva en la eficacia anestésica ${ }^{44-48}$, en tanto que otros señalan que no ${ }^{49-55}$. Posiblemente, en estos estudios, el tipo de anestésico utilizado, el tipo de diente afectado o el sitio de infiltración pudieron haber influido en los resultados.

En cuanto a la preferencia de los pacientes por los dispositivos a presión, un estudio informó $70 \%$ de preferencia para MadaJet XL sobre la técnica tradicional ${ }^{20}$, y en otro se señala una preferencia de $93 \%^{11}$. Sin embargo, en un estudio obtuvo solo un $33.3 \%$ de preferencia al compararlo con la crema EMLA, por el aspecto voluminoso de este dispositivo ${ }^{19}$. En otros estudios en los que se utilizó INJEX, la preferencia disminuye ampliamente, entre $17.6 \%$ y $12.6 \%^{8,17}$, en tanto que en otro alcanza el $77.7 \%^{22}$ . Comfort in, no mostró diferencias significativas con la técnica tradicional ${ }^{24}$. Se ha citado que género y edad no se relacionan con la preferencia de los pacientes ${ }^{16,17}$. Como el dolor es una entidad multidimensional, los factores fisiológicos, económicos, legales, éticos y sociológicos ${ }^{56}$ pueden afectar su percepción. Muchos estudios han demostrado que la percepción del dolor depende de la variabilidad individual en los umbrales relacionados con el género, ${ }^{57,60}$ etnia, ${ }^{61,65}$ edad, ${ }^{66,68}$ e incluso el ritmo circadiano, ${ }^{69,70}$ factores que posiblemente afectaron a la percepción del dolor de los pacientes estudiados y por tanto su preferencia por alguna de las técnicas.

Respecto a la duración del efecto anestésico, todos los estudios concuerdan en que la técnica tradicional tiene una duración significativamente mayor que la técnica libre de aguja. Un estudio informa una duración de $14.00 \pm 9.24 \mathrm{~min}$ para los dispositivos a presión vs. $21.71 \pm 4.63 \mathrm{~min}$ para la jeringa tradicional, ${ }^{8}$ en otro se reporta $20,75 \pm 3,53 \mathrm{~min}$ (técnica a presión) vs. $50 \pm 9.32$ min (técnica tradicional), ${ }^{20}$ en tanto que otros autores han encontrado una duración anestésica de 20 min para la técnica a presión y 40 min para la técnica tradicional. ${ }^{24}$

La mayor duración del efecto anestésico con el sistema tradicional respecto a los dispositivos a presión, puede atribuirse a los procesos farmacocinéticos que ocurren durante la difusión del anestésico ${ }^{1}$. Una inyección a presión que deposita una alta concentración anestésica en una fracción de segundo, posiblemente permite una mayor tasa de difusión desde el espacio extraneural hacia el interior del nervio, esta difusión continúa hasta que existe un equilibrio, después de lo cual la anestesia disminuye a medida que las moléculas comienzan a extenderse fuera del nervio. ${ }^{1}$

Cuando los fascículos del manto comienzan a perder el anestésico local, el anestésico presente en las fibras nerviosas centrales se difunde hacia el manto, por lo que son las primeras en perder la anestesia por completo. Los impulsos nerviosos dolorosos permanecen bloqueados solo mientras 
el anestésico local se encuentra dentro del nervio, y este período define la duración de la anestesia. Por consiguiente, el aumento en la velocidad de todos estos eventos debido a la alta concentración inicial de la solución anestésica después de la inyección a presión en comparación con la infiltración lenta con una jeringa tradicional, podría explicar la diferencia significativa en la duración de la anestesia pulpar. ${ }^{1}$

Se debe considerar que, al utilizar un sistema a presión, la solución anestésica se debe combinar con un agente vasoconstrictor. Esto fue demostrado por un estudio que comparó lidocaína al 2\% más epinefrina 1:80 000 vs mepivacaína al 3\%, cuando se utilizó el dispositivo a presión que sólo contenía mepivacaína en ninguno de los pacientes se logró anestesia pulpar. La insuficiencia de una solución sin vasoconstrictor para lograr anestesia pulpar se explica por la acelerada absorción que se produce después de su rápida aplicación bajo presión; como no hay acción vasoconstrictora, entra rápidamente a la circulación general. ${ }^{8}$

Por otro lado, en un estudio en el cual se analizó diferentes diámetros de boquilla, creados mediante láser en el dispositivo INJEX, que oscilaron entre 0,17 hasta 0,50 $\mathrm{mm}$, se determinó que el diámetro desempeña un papel importante $^{15}$. Así, la profundidad de inyección aumenta a medida que el diámetro de la boquilla se incrementó, en consecuencia, es fácil estimular nervios profundos, causando dolor adicional. La duración y presión de la inyección disminuyeron, si el diámetro de la boquilla aumenta. Cuando el diámetro de la boquilla era $0,30 \mathrm{~mm}$, se aseguró una adecuada profundidad, dispersión y absorción de la inyección, se evitó un trauma excesivo al tejido y, además, se acortó efectivamente el tiempo de inyección. Por lo tanto, un diámetro de boquilla de $0,30 \mathrm{~mm}$ proporcionaría una experiencia favorable y eficiente, tanto para el profesional como para el paciente, factor que debería considerarse para futuros diseños de estos dispositivos. ${ }^{15}$

Respecto a Kovanaze, a pesar de que ha sido aprobado desde el año 2016, un estudio demuestra que el conocimiento es insuficiente en la mayoría de profesionales, ya que desconocen la técnica de administración, duración de acción, biodisponibilidad, número de aplicaciones necesarias, región a anestesiarse y, únicamente el $57 \%$ de los encuestados ha escuchado sobre esta técnica. ${ }^{31}$

La mayoría de los estudios realizados reportan la eficacia de kovanaze para procedimientos restaurativos en dientes anterosuperiores. Como lo muestra un estudio realizado en 150 participantes, en donde el $88 \%$ de los que recibieron Kovanaze pudieron completar el procedimiento dental restaurativo sin la necesidad de anestesia de rescate, en comparación con el $28 \%$ de los pacientes que recibieron placebo, además, reportó una disminución del éxito anestésico entre 60 y $66 \%$ para segundos premolares con Kovanaze $^{37}$. En otro estudio de 110 pacientes en donde, 44 de ellos recibieron kovanaze intranasal, 44 tetracaína sola intranasal y 22 placebo, también se confirmó la eficacia anestésica del $84.1 \%$ para Kovanaze, que permitió terminar el procedimiento restaurativo en dientes anteriores y premolares superiores, en comparación con el $27.3 \%$ que obtuvieron los grupos de tetracaína sola y placebo. ${ }^{6}$

Así mismo, otro estudio comparó la eficacia y seguridad de Kovanaze en 45 adultos sanos, en el que 15 recibieron pulverización intranasal con Kovanaze y luego inyección simulada, otros 15 recibieron primero inyección simulada y luego spray Kovanaze, 8 inyección de un cartucho lidocaína al $2 \%$ con epinefrina 1:100,00 y luego solución salina tamponada intranasal $\mathrm{y}, 7$ recibieron solución salina tamponada intranasal y a continuación inyección de lidocaína. El $83.3 \%$ de los pacientes que recibieron Kovanaze, así como el $93.3 \%$ que recibieron lidocaína inyectada, no requirió anestesia de rescate para completar los procedimientos dentales restaurativos, confirmando su éxito anestésico y, ambos tipos de administración anestésica fueron bien tolerados. ${ }^{33}$

Cabe destacar que la aprobación de Kovanaze intranasal por parte de la FDA, se basó especialmente en la superioridad respeto al placebo ${ }^{33}$, lo cual confirma un reciente estudio en el que 50 pacientes que recibieron 2 tipos diferentes de administración anestésica en 2 citas separadas por 1 semana. En la primera cita se administró Kovanaze e infiltración inyectable de lidocaína simulada, en tanto que en la segunda cita recibieron 2 administraciones intranasales de placebo y una infiltración $1.8 \mathrm{ml}$ de lidocaína al $2 \%$ con epinefrina al 1: 100,000; la anestesia pulpar fue significativamente menos exitosa con Kovanaze intranasal e infiltración simulada entre 22 y $37 \%$ comparada con el 89 y $91 \%$ para aerosol intranasal simulado y la infiltración inyectable de lidocaína. ${ }^{30}$

En el mismo estudio se evaluó la preferencia ante la técnica de anestesia local inyectable con lidocaína al $2 \%$, en el cual, $56 \%$ de los pacientes, antes de participar en la investigación, prefirió el aerosol intranasal, pero después de experimentar las 2 vías de administración, el $100 \%$ prefirió la técnica anestésica convencional, ya que informaron mayor molestia y dolor con Kovanaze. ${ }^{30}$

El éxito de la técnica convencional con aguja puede atribuirse a que el líquido anestésico se deposita muy cerca a los ápices dentales, por lo que recorre una distancia muy corta para llegar a los plexos nerviosos, a diferencia de la aplicación anestésica intranasal que ingresa a la corteza ósea del seno maxilar y desde allí la distancia a los ápices de primeros premolares es alrededor de 8-9 $\mathrm{mm}$, por lo que mientras más posterior, menor es la eficacia anestésica ${ }^{30}$. 
Finalmente, podríamos preguntarnos si la técnica tradicional con aguja constituye el estándar de oro (gold standard) o el estándar de atención (standard of care). Debe tenerse en cuenta que estos términos no son sinónimos ni intercambiables. El estándar de oro se define como "algo establecido como un ejemplo contra el cual se comparan otros del mismo tipo", 71 mientras que estándar de atención es definido como "el grado de cuidado o competencia que se espera ejercer en una circunstancia particular". ${ }^{72}$ En base a lo expuesto, la técnica con aguja continúa siendo el estándar de oro en la aplicación de técnicas anestésicas, sin embargo, existe flexibilidad en la toma de decisiones clínicas en una variedad de entornos de atención al paciente, ${ }^{73}$ que constituirían el estándar de atención; por ejemplo, cuando un paciente tiene tripanofobia, las técnicas sin aguja son adecuadas, pero no cuando se requiere un bloqueo nervioso. Así la toma de decisiones clínicas del profesional dependería de cada caso clínico en particular. Por otro lado, si una técnica ofrece beneficios sobre las técnicas tradicionales o es la "más usada" en cierto período de tiempo, ello no implica que por ese hecho pueda ser definida como el estándar de atención. ${ }^{74}$

El estándar de atención es una obligación ética; es fundamental y es clave para una atención de calidad y excelencia en odontología, que cambia constantemente, ya que cada caso clínico es único y la ciencia, las opciones de tratamiento y las tecnologías están en constante evolución. Sin embargo, el conocimiento obtenido de los últimos avances no implica que su uso deba ser obligatorio en todos los casos; más bien, el dentista debe ser prudente y competente en la adopción de estos nuevos métodos o técnicas. ${ }^{74}$ Muchas ïnnovaciones"no cumplen las expectativas establecidas por sus creadores y desaparecen o siguen siendo técnicas o dispositivos complementarios ${ }^{1}$ . En este sentido, la desimplementación se ha propuesto como práctica de abandono y desaprendizaje de técnicas o métodos que son no comprobadas (sin evidencia o validación) ó habituales, que continúan realizándose a pesar de la evidencia contradictoria. ${ }^{75}$

\section{Conclusiones}

Hasta la fecha, la efectividad de las técnicas descritas en esta revisión ha sido reportada como limitada y, al parecer, mientras más complejo y de larga duración es el procedimiento odontológico, menor es el éxito. Si bien podrían ser una buena alternativa para generar anestesia local y evitar ciertos riesgos que causan las inyecciones con aguja, estas técnicas no han superado al método de anestesia dental convencional, por lo que el odontólogo deberá tomar en cuenta el grado de miedo o ansiedad del paciente a las agujas, así como también la relación costo- beneficio, antes de emplear estas técnicas en su consulta.

\section{Perspectivas futuras}

Aunque algunas de las técnicas de anestesia dental sin aguja están disponibles desde hace años atrás, la escasez de estudios evita posiblemente su auge, por lo que se recomienda realizar más investigaciones para establecer con claridad su efectividad, especialmente en procedimientos más invasivos. Así mismo, se recomienda la inclusión de pacientes con tripanofobia en futuros estudios, en quienes sería de mayor utilidad. Futuras investigaciones podrían permitir superar las limitaciones y desventajas inherentes a las técnicas mencionadas, sobre todo volumen administrado, dificultad de conseguir anestesia pulpar y el hecho de no ser útiles en anestesia dental troncular. Además, se podrían implementar técnicas o vías de administración sin aguja nuevas o que complementen a las actuales.

Una ciencia emergente que podría aportar al desarrollo de nuevas metodologías en el control y modulación del dolor es la optogenética, la cual es una técnica biológica, aplicada por ahora in vitro y en animales de experimentación, que emplea luz para controlar células, particularmente neuronas, que han sido modificadas genéticamente para expresar canales iónicos sensibles a la luz, resultando una neuromodulación mediante medios ópticos y genéticos. ${ }^{1,76}$

Además, mediante el empleo de fotofarmacología (sin realizar modificaciones genéticas) se ha desarrollado un nuevo tipo de anestésico local que puede ser "encendido y apagado" mediante el uso de luz de diferentes longitudes de onda; esta nueva molécula es fotoisomerizable, químicamente se parece a la lidocaína y, es el amonio cuaternario-azobenceno-amonio cuaternario (QAQ, por sus siglas en inglés). ${ }^{77,78}$ Esta técnica clínicamente permitiría la consecución espaciotemporal de la anestesia, esto es, en un sitio específico y de la duración deseada, variando la longitud de onda o la intensidad de la luz. ${ }^{1}$

El QAQ existe en dos estados, cis y trans. En su forma activa, trans, la molécula es recta, pero la exposición a luz de $380 \mathrm{~nm}$ la convierte en su forma cis, que tiene forma de una L; la aplicación de luz de $500 \mathrm{~nm}$ rápidamente revierte la forma cis a trans. Una vez dentro de la célula, en su forma trans el QAQ bloquea varios canales iónicos diferentes y es selectivo para neuronas sensibles al dolor, ${ }^{1,78}$ por lo que permite bloquear la nocicepción sin afectar axones motores u otras sensaciones $^{79}$. Se ha demostrado que el QAQ bloquea canales de sodio y potasio dependientes de voltaje y canales de calcio. ${ }^{77,78}$ Sin embargo, estos estudios se han realizado utilizando solo modelos in vitro. ${ }^{77,78}$

Aunque su desarrollo es incipiente, la optogenética y los compuestos activados-inactivados con luz (fotoswitches) son 
prometedores medios para el manejo perioperatorio del do- 13 lor $^{1}$, y podrían mejorar o suplementar las técnicas anestésicas con o sin aguja en un futuro no tan lejano.

\section{Agradecimiento}

Nos gustaría agradecer a André Peralta por el diseño de todas las figuras incluidas en la presente revisión. Este trabajo se basa en un requisito de cumplimiento de grado para que Jessica Álvarez y Andrea Ruiz obtengan el título de odontólogo. El estudio fue autofinanciado por los autores.

Conflictos de interés: Los autores declaran que no tienen ningún conflicto de interés.

\section{Referencias}

1 Malamed S. Handbook of local anesthesia. 7th ed. St. Louis, MO: Elsevier; 2019.

2 Kaufman E, Epstein J, Naveh E, Gorsky M, Gross A, Cohen G. A survey of pain, pressure, and discomfort induced by commonly used oral local anesthesia injections. Anesth Prog. 2005; 52: 122-7.

3 Hamilton J. Needle phobia: a neglected diagnosis. J Fam Pract. 1995; 41: 169-75.

4 Dionne R, Gordon S, Mccullagh L, Phero J. Assessing the need for anesthesia and sedation in the general population. J Am Dent Assoc. 1998; 129: 167-73.

5 Blanton P, Jeske A. Avoiding complications in local anesthesia induction: anatomical considerations. J Am Dent Assoc. 2003; 134: 888-93.

6 Ciancio S, Marberger A, Ayoub F, Garlapo D, Pantera E, Pantera C, et al. Comparison of 3 intranasal mists for anesthetizing maxillary teeth in adults: A randomized, double-masked, multicenter phase 3 clinical trial. J Am Dent Assoc. 2016; 147: 339-47.

7 Whittemore R, Knafl K. The integrative review: updated methodology. J Adv Nurs. 2005; 52: 546-53.

8 Dabarakis N, Alexander V, Tsirlis A, Parissis N, Nikolaos M. Needle-less local anesthesia: clinical evaluation of the effectiveness of the jet anesthesia Injex in local anesthesia in dentistry. Quintessence Int. 2007; 38: E572-576.

9 Margetis P, Quarantillo E, Lindberg R. Jet injection local anesthesia in dentistry: a report of 66 cases. U S Armed Forces Med J. 1958; 9: 625-34.+

10 Taberner A, Hogan N, Hunter I. Needle-free jet injection using real-time controlled linear Lorentz-force actuators. Med Eng Phys. 2012; 34: 1228-35.

11 Munshi A, Hegde A, Bashir N. Clinical evaluation of the efficacy of anesthesia and patient preference using the needleless jet syringe in pediatric dental practice. J Clin Pediatr Dent. 2001; 25: 131-6.

12 Kale T, Momin M. Needle free injection technology - An overview. Farm Syst Health. 2014; 5: 1-8
3 Schramm-Baxter J, Mitragotri S. Needle-free jet injections: dependence of jet penetration and dispersion in the skin on jet power. J Control Release. 2004; 97: 527-35.

14 Grant T, Stockwell K, Morrison J, Mann Dd Effect of injection pressure and fluid volume and density on the jet dispersion pattern of needle-free injection devices. Biosyst Eng. 2015; 138: 59-64.

15 Zeng D, Kang Y, Xie L, Xia X, Wang Z, Liu W. A Mathematical Model and Experimental Verification of Optimal Nozzle Diameter in Needle-Free Injection. J Pharm Sci. 2018; 107: 1086-94.

16 Ocak H, Akkoyun EF, Çolpak HA, Demetoglu U, Yücesoy $\mathrm{T}$, Kilic E, et al. Is the jet injection effective for teeth extraction?. Stomatol Oral Maxillo Fac. 2019; 700: 1-6.

17 Arapostathis K, Dabarakis N, Coolidge T, Tsirlis A, Kotsanos N. Comparison of Acceptance, Preference, and Efficacy Between Jet Injection INJEX and Local Infiltration Anesthesia in 6 to 11 Year Old Dental Patients. Anesth Prog. 2010; 57: 3-12.

18 Barolet D, Benohanian A. Current trends in needle-free jet injection: an update. Clin Cosmet Investing Dermatol. 2018; 11: $231-8$.

19 Gupta R, Kaur S, Dahiya P, Kumar M. Comparative evaluation of efficacy of EMLA and needleless jet anesthesia in non-surgical periodontal therapy. J Oral Biol Craniofac Res. 2018; 8: 118-21.

20 Makade C, Shenoi P, Gunwal M. Comparison of acceptance, preference and efficacy between pressure anesthesia and classical needle infiltration anesthesia for dental restorative procedures in adult patients. J Conserv Dent. 2014; 17: 169.

21 Mika Medical Co (2019). Comfort-in Needle Free Injection System. Introductions for use. [WWW document]. URLhttps://www.injectneedlefree.com/user-manuals/ [accessed on 20 October 2019].

22 Kumar D, Agrawal A, Gupta H, Gupta S, Mehra H, Singh J. Needle-less injectors: clinical evaluation of effectiveness of jet injector as local infiltration anesthesia for dental extractions. Int J Sci Res. 2019; 8: 11-15.

23 Ogle O, Mahjoubi G. Advances in Local Anesthesia in Dentistry. Dent Clin N Am. 2011; 55: 481-99.

24 Oliveira A, Amorim K, Nascimento Júnior E, Duarte A, Groppo F, Takeshita W, et al. Assessment of anesthetic properties and pain during needleless jet injection anesthesia: a randomized clinical trial. J Appl Oral Sci. 2019; 27: 1-7.

25 Angelo Z, Polyvios C. Alternative practices of achieving anaesthesia for dental procedures: a review. J Dent Anesth Pain Med. 2018; 18: 79-88.

26 Graham J. Profound, needle-free anesthesia in orthodontics. J Clin Orthod. 2006; 40: 723-4.

27 Kumar S. Newer delivery systems for local anesthesia in dentistry. Int J Pharm Sci Res. 2015; 7: 252-5.

28 Boyce Ra, Kirpalani T, Mohan N. Updates of Topical and Local Anesthesia Agents. Dent Clin N Am. 2016; 60: 445-71. 
29 US Food And Drug Administration (2016). Kovanaze. Available from: URL:https://www.accessdata.fda.gov/scripts/cder/daf/index. $\mathrm{cfm}$ ?event=overview.process \&ApplNo=208032 [Accessed 42 on 23 September 2019].

30 Capetillo J, Drum M, Reader A, Fowler S, Nusstein J, Beck M. Anesthetic Efficacy of Intranasal 3\% Tetracaine plus $0.05 \%$ Oxymetazoline (Kovanaze) in Maxillary Teeth. J 43 Endod. 2019; 45: 257-62.

31 Kumar A, Raj J. Knowledge, attitude, and practices regarding the use of nasal spray anesthesia by dental practitioners. Drug invent today. 2018; 10: 2023-28.

32 Hersh E, Saraghi M, Moore P. Intranasal tetracaine and oxymetazoline: a newly approved drug formulation that 45 provides maxillary dental anesthesia without needles. Curr Med Res Opin. 2016; 32: 1919-25.

33 Ciancio S, Hutcheson M, Ayoub F, Pantera E, Pantera C, Garlapo D, et al. Safety and efficacy of a novel nasal spray 46 for maxillary dental anesthesia. J Dent Res. 2013; 92: 43S$8 \mathrm{~S}$.

34 Hersh E, Saraghi M, Moore P. Two Recent Advances in Local Anesthesia: Intranasal Tetracaine/Oxymetazoline and 47 Liposomal Bupivacaine. Curr Oral Health Rep. 2017; 4: 189-196.

35 Giannakopoulos H, Levin L, Chou J, Cacek A, Hutcheson M, Secreto S, et al. The cardiovascular effects and pharmacokinetics of intranasal tetracaine plus oxymetazoline: preliminary findings. J Am Dent Assoc. 2012; 143: 872-80.

36 Higgins T, Hwang P, Kingdom T, Orlandi R, Stammberger H, Han J. Systematic Review of Topical Vasoconstrictors in Endoscopic Sinus Surgery. Laryngoscope. 2011; 121: 49 422-32.

37 Hersh E, Pinto A, Saraghi M, Saleh N, Pulaski L, Gordon $\mathrm{S}$, et al. Double-masked, randomized, placebo-controlled study to evaluate the efficacy and tolerability of intranasal K305 (3\% tetracaine plus $0.05 \%$ oxymetazoline) in 50 anesthetizing maxillary teeth. J Am Dent Assoc. 2016; 147: 278-87.

38 Cacek A, Gobburu J, Gopalakrishnan M. Population Pharmacokinetics of an Intranasally Administered Combination of Oxymetazoline and Tetracaine in Healthy Volunteers. J Clin Pharmacol. 2017; 57: 247-54.

39 Misra A, Shahiwala A. Novel drug delivery technologies in- 5 novative strategies for drug re-positioning, 1st ed. Springer Singapore, 2019.

40 Laube B, Sharpless G, Vikani A, Harrand V, Zinreich S, Sedberry K, et al. Intranasal Deposition of AccusprayTM 5 Aerosol in Anatomically Correct Models of 2-, 5-, and 12Year-Old Children. J Aerosol Med Pulm Drug Deliv. 2015; 28: 320-33.

41 Evans G, Yiming L. A phase 3, multi-center, randomized, double-blind, parallel-groups clinical trial comparing the efficacy and safety of intranasally administered K-305 to placebo for anesthetizing maxillary teeth in pediatric patients.
2016 [citado 2 de abril de 2020]. Available from:URL: https://www.fda.gov/downloads/Drugs/Development ApprovalProcess/DevelopmentResources/UCM513665.pdf 2 Madurantakam P. Kovanaze Vs. Articaine in Achieving Pulpal Anesthesia of Maxillary Teeth - Pediatric. 2019 [citado 2 de abril de 2020]. Available from: URL: https://clinicaltrials.gov/ct2/show/NCT03962634

43 Szmuk P, Szmuk E, Ezri T. Use of needle-free injection systems to alleviate needle phobia and pain at injection. Expert Rev Pharmacoenomics Outcomes Res. 2005; 5: 467-77.

44 Milani A, Froughreyhani M, Rahimi S, Zand V, Jafarabadi Ma. Volume of Anesthetic Agents and IANB Success: A Systematic Review. Anesth Prog. 2018; 65: 16-23.

45 Abazarpoor R, Parirokh M, Nakhaee N, Abbott P. A Comparison of Different Volumes of Articaine for Inferior Alveolar Nerve Block for Molar Teeth with Symptomatic Irreversible Pulpitis. J Endod. 2015; 41: 1408-11.

6 Mikesell A, Drum M, Reader A, Beck M. Anesthetic Efficacy of $1.8 \mathrm{~mL}$ and $3.6 \mathrm{~mL}$ of $2 \%$ Lidocaine with 1:100,000 Epinephrine for Maxillary Infiltrations. J Endod. 2008; 34: 121-5.

7 Aggarwal V, Singla M, Miglani S, Kohli S, Sharma V, Bhasin S. Does the volume of supplemental intraligamentary injections affect the anaesthetic success rate after a failed primary inferior alveolar nerve block? A randomizeddouble blind clinical trial. Int Endod J. 2018; 51: 5-11.

48 Brunetto P, Ranali J, Ambrosano G, De Oliveira P, Groppo F, Meechan J, et al. Anesthetic efficacy of 3 volumes of lidocaine with epinephrine in maxillary infiltration anesthesia. Anesth Prog. 2008; 55: 29-34.

49 Singla M, Subbiya A, Aggarwal V, Vivekanandhan P, Yadav $\mathrm{S}$, Yadav $\mathrm{H}$, et al. Comparison of the anaesthetic efficacy of different volumes of $4 \%$ articaine $(1.8$ and $3.6 \mathrm{~mL})$ as supplemental buccal infiltration after failed inferior alveolar nerve block. Int Endod J. 2015; 48: 103-8.

50 Pfeil L, Drum M, Reader A, Gilles J, Nusstein J. Anesthetic Efficacy of 1.8 Milliliters and 3.6 Milliliters of $2 \%$ Lidocaine with 1:100,000 Epinephrine for Posterior Superior Alveolar VREELAND DL, READER A, BECK M, MEYERS W, WEAVER J. An evaluation of volumes and concentrations of lidocaine in human inferior alveolar nerve block. J Endod. 1989; 15: 6-12.

51 Vreeland D, Reader A, Beck M, Meyers W, Weaver J. An evaluation of volumes and concentrations of lidocaine in human inferior alveolar nerve block. J Endod. 1989; 15: 6-12.

52 Nusstein J, Reader A, Beck F. Anesthetic efficacy of different volumes of lidocaine with epinephrine for inferior alveolar nerve blocks. Gen Dent. 2002; 50: 372-5.

53 Parirokh M, Satvati S, Sharifi R, Rekabi A, Gorjestani H, Nakhaee N, et al. Efficacy of combining a buccal infiltration with an inferior alveolar nerve block for mandibular molars with irreversible pulpitis. Oral Surg, Oral Med, Oral Pathol, Oral Radiol, and Endod. 2010; 109: 468-73. 
54 Wali M, Drum M, Reader A, Nusstein J. Prospective, Randomized Single-blind Study of the Anesthetic Efficacy of 1.8 and 3.6 Milliliters of 2\% Lidocaine with 1:50,000 71 Epinephrine for Inferior Alveolar Nerve Block. J Endod. 2010; 36: 1459-62.

55 Fowler S, Reader A. Is a Volume of $3.6 \mathrm{~mL}$ Better than $1.8 \mathrm{~mL}$ for Inferior Alveolar Nerve Blocks in Patients 72 with Symptomatic Irreversible Pulpitis? J Endod. 2013; 39: 970-2.

56 Ballantyne J, Fishman S, Rathmell J. Bonica's management of pain, 5th ed. Philadelphia: Wolters Kluwer, 2019.

57 Chesterton L, Barlas P, Foster N, Baxter D, Wright C. Gender differences in pressure pain threshold in healthy humans: Pain. 2003; 101: 259-66.

58 Defrin R, Shramm L, Eli I. Gender role expectations of pain is associated with pain tolerance limit but not with pain 75 threshold: Pain. 2009; 145: 230-6.

59 Martin R. Influence of biological sex, trait gender, and state gender on pain threshold, pain tolerance, and ratings of pain 76 severity. Pers Individ Diff. 2019; 138: 183-7.

60 Shaefer J, Khawaja S, Bavia P. Sex, Gender, and Orofacial 77 Pain. Dent Clin N Am. 2018; 62: 665-82.

61 Rahim W, Riley J, Williams A, Fillingim R. A Quantitative Review of Ethnic Group Differences in Experimental Pain Response: Do Biology, Psychology, and Culture Matter?. 78 Pain Med. 2012; 13: 522-40.

62 Ono K, Viet C, Ye Y, Dang D, Hitomi S, Toyono T, et al. Cutaneous pigmentation modulates skin sensitivity via tyrosinase-dependent dopaminergic signalling. Sci Rep. 2017; 7: 9181.

63 Campbell C, Edwards R. Ethnic differences in pain and pain management. Pain Manage. 2012; 2: 219-30.

64 Komiyama O, Kawara M, De Laat A. Ethnic Differences Regarding Tactile and Pain Thresholds in the Trigeminal Region. J Pain. 2007; 8: 363-9.

65 Vigil J, Coulombe P, Rowell L, Strenth C, Kruger E, Alcock J, et al. The Confounding Effect of Assessor Ethnicity on Subjective Pain Reporting in Women. TOATJ. 2017; 11: $1-11$.

66 Lautenbacher S, Peters Jh, Heesen M, Scheel J, Kunz M. Age changes in pain perception: A systematic-review and meta-analysis of age effects on pain and tolerance thresholds. Neurosci Biobehav Rev. 2017; 75: 104-13.

67 El Tumi H, Johnson M, Dantas P, Maynard M, Tashani O. Age-related changes in pain sensitivity in healthy humans: A systematic review with meta-analysis. Eur J Pain. 2017; 21: 955-64.

68 Petrini L, Matthiesen S, Arendt-Nielsen L. The Effect of Age and Gender on Pressure Pain Thresholds and Suprathreshold Stimuli. Perception. 2015; 44: 587-96.

69 Crodelle J, Piltz S, Hagenauer M, Booth V. Modeling the daily rhythm of human pain processing in the dorsal horn. PLOS Comput Biol. 2019; 15: 1-23.

70 Kim M, Chung J, Kho H, Park J. The Circadian Rhythm
Variation of Pain in the Orofacial Region. J Oral Med Pain. 2015; 40: 89-95.

1 Merriam-Webster.com Thesaurus, Merriam-Webster,. "Gold standard". [citado 24 de febrero de 2020]. Available from: URL: https://www.merriamwebster.com/thesaurus/gold \%20standard

72 Merriam-Webster.com Legal Dictionary, MerriamWebster, "Standard of care." [citado 24 de febrero de 2020]. Available from: URL: https://www.merriamwebster.com/legal/standard \%20of \%20care

73 Hardman H, Smyth T, Semo J, D'ercole F. UltrasoundGuided Regional Anesthesia and Standard of Care. Reg Anesth Pain Med. 2018; 43: 107.

74 Gokul S, Prithviraj K. Standard of care in dentistry. J Orofac Sci. 2012; 4: 100-2.

75 Prasad V, Ioannidis J. Evidence-based de-implementation for contradicted, unproven, and aspiring healthcare practices. Implement Sci. 2014; 9: 1-5.

6 Eliot S, Hunter P, Ali R. Neuromodulation, 2nd ed. St. Louis, MO: Elsevier; 2018.

Mourot A., Herold C., Kienzler M., Kramer R. Understanding and improving photo-control of ion channels in nociceptors with azobenzene photo-switches. Br J Pharmacol. 2018; 175: 2296-331.

78 Mourot A, Fehrentz T, Le Feuvre Y, Smith C, Herold C, Dalkara D, et al. Rapid optical control of nociception with an ion-channel photoswitch. Nat Methods. 2012; 9: 396-402.

79 Mourot A, Tochitsky I, Kramer R. Light at the end of the channel: optical manipulation of intrinsic neuronal excitability with chemical photoswitches. Front Mol Neurosci. 2013; 6: 1-15.

Recibido: 30 de septiembre de 2020

Aceptado: 01 de diciembre de 2020 
